\title{
Research Article Schur-Convexity of Averages of Convex Functions
}

\author{
Vera Čuljak, ${ }^{1}$ Iva Franjić, ${ }_{r}^{2}$ Roqia Ghulam, ${ }^{3}$ and Josip Pečarić ${ }^{4}$ \\ ${ }^{1}$ Department of Mathematics, Faculty of Civil Engineering, University of Zagreb, Kačićeva 26, \\ 10000 Zagreb, Croatia \\ ${ }^{2}$ Faculty of Food Technology and Biotechnology, University of Zagreb, Pierottijeva 6, 10000 Zagreb, Croatia \\ ${ }^{3}$ Abdus Salam School of Mathematical Sciences, 68-B, New Muslim Town, Lahore 54600, Pakistan \\ ${ }^{4}$ Faculty of Textile Technology, University of Zagreb, Prilaz baruna Filipovića 28a, 10000 Zagreb, Croatia
}

Correspondence should be addressed to Roqia Ghulam, rukiyya@gmail.com

Received 12 November 2010; Accepted 11 January 2011

Academic Editor: Matti K. Vuorinen

Copyright (C 2011 Vera Čuljak et al. This is an open access article distributed under the Creative Commons Attribution License, which permits unrestricted use, distribution, and reproduction in any medium, provided the original work is properly cited.

The object is to give an overview of the study of Schur-convexity of various means and functions and to contribute to the subject with some new results. First, Schur-convexity of the generalized integral and weighted integral quasiarithmetic mean is studied. Relation to some already published results is established, and some applications of the extended result are given. Furthermore, Schur-convexity of functions connected to the Hermite-Hadamard inequality is investigated. Finally, some results on convexity and Schur-convexity involving divided difference are considered.

\section{Introduction}

The property of Schur-convexity and Schur-concavity has invoked the interest of many researchers and numerous papers have been dedicated to the investigation of it. The object of this paper is to present an overview of the results related to the study of Schur-convexity of various means and functions, in particular, those connected with the Hermite-Hadamard inequality. Moreover, we contribute to the subject with some new results.

First, let us recall the definition of Schur-convexity. It generalizes the definition of the convex and concave function via the notion of majorization.

Definition 1.1. Function $F: A \subseteq \mathbb{R}^{n} \rightarrow \mathbb{R}$ is said to be Schur-convex on $A$ if

$$
F\left(x_{1}, x_{2}, \ldots, x_{n}\right) \leq F\left(y_{1}, y_{2}, \ldots, y_{n}\right)
$$


for every $\mathbf{x}=\left(x_{1}, x_{2}, \ldots, x_{n}\right), \mathbf{y}=\left(y_{1}, y_{2}, \ldots, y_{n}\right) \in A$ such that $\mathbf{x} \prec \mathbf{y}$, that is, such that

$$
\sum_{i=1}^{k} x_{[i]} \leq \sum_{i=1}^{k} y_{[i]}, \quad k=1,2, \ldots, n-1, \quad \sum_{i=1}^{n} x_{[i]}=\sum_{i=1}^{n} y_{[i]}
$$

where $x_{[i]}$ denotes the $i$ th largest component in $\mathbf{x}$.

Function $F$ is said to be Schur-concave on $A$ if $-F$ is Schur-convex.

Note that every function that is convex and symmetric is also Schur-convex.

One of the references which will be of particular interest in this paper is [1]. The authors were inspired by some inequalities concerning gamma and digamma function and proved the following result for the integral arithmetic mean.

Theorem A1. Let $f$ be a continuous function on an interval I with a nonempty interior. Then,

$$
F(x, y)= \begin{cases}\frac{1}{y-x} \int_{x}^{y} f(t) d t, & x, y \in I, x \neq y, \\ f(x), & x=y \in I\end{cases}
$$

is Schur-convex (Schur-concave) on $I^{2}$ if and only if $f$ is convex (concave) on I.

Few years later, Wulbert, in [2], proved that the integral arithmetic mean $F$ defined in (1.3) is convex on $I^{2}$ if $f$ is convex on $I$. Zhang and $\mathrm{Chu}$, in [3], rediscovered (without referring to and citing Wulbert's result) that the necessary and sufficient condition for the convexity of the integral arithmetic mean $F$ is for $f$ to be convex on $I$. Note that the necessity is obvious. Namely, if $F$ is convex, then it is also Schur-convex since it is symmetric. Theorem A1 then implies the convexity of function $f$.

Later, in [4], the Schur-convexity of the weighted integral arithmetic mean was proved.

Theorem A2. Let $f$ be a continuous function on $I \subseteq \mathbb{R}$ and let $p$ be a positive continuous weight on I. Then, the function

$$
F_{p}(x, y)= \begin{cases}\frac{1}{\int_{x}^{y} p(t) d t} \int_{x}^{y} p(t) f(t) d t, & x, y \in I, x \neq y, \\ f(x), & x=y\end{cases}
$$

is Schur-convex (Schur-concave) on $I^{2}$ if and only if the inequality

$$
\frac{\int_{x}^{y} p(t) f(t) d t}{\int_{x}^{y} p(t) d t} \leq \frac{p(x) f(x)+p(y) f(y)}{p(x)+p(y)}
$$

holds (reverses) for all $x, y$ in $I$.

In the same reference, the authors left an open problem: under which conditions does (1.5) hold?

The monotonicity of the function $F_{p}$ defined in (1.4) was studied in [5]. 
Theorem A3. Let $f$ be a continuous function on $I \subseteq \mathbb{R}$ and let $p$ be a positive continuous weight on $I$. Then, the function $F_{p}(x, y)$ defined in (1.4) is increasing (decreasing) on $I^{2}$ if $f$ is increasing (decreasing) on $I$.

In the following sections, Schur-convexity of the generalized integral and weighted integral quasiarithmetic mean is studied. Relation to some already published results is established. Further, a new proof of sufficiency in Theorem A1, which is also a new proof of Wulbert's result from [2], that is, Zhang and Chu's result from [3], is presented. Some applications of this extended result are given. Furthermore, Schur-convexity of various functions connected to the Hermite-Hadamard inequality is investigated. Finally, some results on convexity and Schur-convexity involving divided difference are considered.

To complete the Introduction, we state three very interesting lemmas related to Schurconvexity. They are needed later for the proofs of our results. All three can be found in both $[6,7]$. The first one gives a useful characterization of Schur-convexity.

Lemma A1. Let $I \subset \mathbb{R}$ and let $f: I^{n} \rightarrow \mathbb{R}$ be a continuous symmetric function. If $f$ is differentiable on $I^{n}$, then $f$ is Schur-convex on $I^{n}$ if and only if

$$
\left(x_{i}-x_{j}\right)\left(\frac{\partial f}{\partial x_{i}}-\frac{\partial f}{\partial x_{j}}\right) \geq 0,
$$

for all $x_{i}, x_{j} \in I, i \neq j, i, j=1,2, \ldots, n$. Function $f$ is Schur concave if and only if the reversed inequality sign holds.

Lemma A2. Let $\Phi: \mathbb{R}^{n} \rightarrow \mathbb{R}, g: I \subset \mathbb{R} \rightarrow \mathbb{R}$ and $\Psi: I^{n} \rightarrow \mathbb{R}$ be defined as $\Psi(\mathbf{x})=$ $\Phi\left(g\left(x_{1}\right), \ldots, g\left(x_{n}\right)\right)$, where $\mathbf{x}=\left(x_{1}, \ldots, x_{n}\right)$.

(1) If $g$ is convex (concave) and $\Phi$ is increasing and Schur-convex (Schur-concave), then $\Psi$ is Schur-convex (Schur-concave).

(2) If $g$ is concave (convex) and $\Phi$ is decreasing and Schur-convex (Schur-concave), then $\Psi$ is Schur-convex (Schur-concave).

Lemma A3. Let $\Psi_{i}: A \subset \mathbb{R}^{n} \rightarrow \mathbb{R}, i=1, \ldots, k, h: \mathbb{R}^{k} \rightarrow \mathbb{R}$ and $\Lambda: A \rightarrow \mathbb{R}$ be defined as $\Lambda(\mathbf{x})=h\left(\Psi_{1}(x), \ldots, \Psi_{k}(x)\right)$, where $\mathbf{x}=\left(x_{1}, \ldots, x_{n}\right)$.

(1) If each of $\Psi_{i}$ is Schur-convex and $h$ is increasing (decreasing), then $\Lambda$ is Schur-convex (Schur-concave).

(2) If each of $\Psi_{i}$ is Schur-concave and $h$ is increasing (decreasing), then $\Lambda$ is Schur-concave (Schur-convex).

\section{Generalizations}

Let $p$ be a real positive Lebesgue integrable function on $[a, b], k$ a real Lebesgue integrable function on $[a, b]$, and $f$ a real continuous strictly monotone function defined on $J$, the range 
of $k$. The generalized weighted quasiarithmetic mean of function $k$ with respect to weight function $p$ is given by

$$
M_{f}(p, k ; a, b)=f^{-1}\left(\frac{1}{\int_{a}^{b} p(t) d t} \int_{a}^{b} p(t) f(k(t)) d t\right) .
$$
example,

For a special choice of functions $p, f, k$, we can obtain various integral means. For

(i) for $p(x)=1$ on $[a, b]$, we get the classical quasiarithmetic integral mean of a function $k$

$$
M_{f}(1, k ; a, b)=f^{-1}\left(\frac{1}{b-a} \int_{a}^{b} f(k(t)) d t\right),
$$

(ii) for $k(x)=x=i d(x)$ on $[a, b]$, we get the classical weighted quasiarithmetic integral mean

$$
M_{f}(p, i d ; a, b)=f^{-1}\left(\frac{1}{\int_{a}^{b} p(t) d t} \int_{a}^{b} p(t) f(t) d t\right)
$$

(iii) for $f(x)=x=i d(x)$ on $J$, we get the weighted arithmetic integral mean

$$
M_{i d}(p, k ; a, b)=\frac{1}{\int_{a}^{b} p(t) d t} \int_{a}^{b} p(t) k(t) d t,
$$

(iv) for $f(x)=x^{r}$ on $J$, we obtain the weighted power integral mean of order $r$

$$
M^{[r]}(p, k ; a, b)= \begin{cases}\left(\frac{1}{\int_{a}^{b} p(t) d t} \int_{a}^{b} p(t) k^{r}(t) d t\right)^{1 / r}, & r \neq 0, \\ \exp \left(\frac{1}{\int_{a}^{b} p(t) d t} \int_{a}^{b} p(t) \ln k(t) d t\right), & r=0 .\end{cases}
$$

The next result discovers the property of Schur-convexity of the generalized integral quasiarithmetic means.

Theorem 2.1. Let $k$ be a real Lebesgue integrable function defined on the interval $I \subset \mathbb{R}$, with range $J$. Let $f$ be a real continuous strictly monotone function on $J$. Then, for the generalized integral quasiarithmetic mean of function $k$ defined as

$$
M_{f}(k ; x, y)=f^{-1}\left(\frac{1}{y-x} \int_{x}^{y}(f \circ k)(t) d t\right), \quad(x, y) \in I^{2},
$$


the following hold:

(i) $M_{f}(k ; x, y)$ is Schur-convex on $I^{2}$

if $f \circ k$ is convex on $I$ and $f$ is increasing on $J$

or if $f \circ k$ is concave on $I$ and $f$ is decreasing on $J$,

(ii) $M_{f}(k ; x, y)$ is Schur-concave on $I^{2}$

if $f \circ k$ is convex on $I$ and $f$ is decreasing on $J$

or if $f \circ k$ is concave on $I$ and $f$ is increasing on $J$.

Proof. Applying Theorem A1 for function $f \circ k$ yields that

$$
\Phi(x, y)=\frac{1}{y-x} \int_{x}^{y}(f \circ k)(t) d t
$$

is Schur-convex (Schur-concave) if and only if $f \circ k$ is convex (concave). Now, from Lemma A3 applied for $M_{f}(k ; x, y)=f^{-1}(\Phi(x, y))$, the statement follows.

Remark 2.2. Applying this theorem for $f(t)=t^{r-1}$ and $k(t)=t=i d(t)$ shows that the generalized logarithmic mean defined for $x, y>0$ as

$$
L_{r}(x, y)= \begin{cases}\left(\frac{y^{r}-x^{r}}{r(y-x)}\right)^{1 /(r-1)}, & r \in \mathbb{R} \backslash\{0,1\}, x \neq y, \\ \frac{1}{e}\left(\frac{x^{x}}{y^{y}}\right)^{1 /(x-y)}, & r=1, x \neq y, \\ \frac{y-x}{\log y-\log x}, & r=0, x \neq y, \\ x, & r \in \mathbb{R}, x=y\end{cases}
$$

is Schur-convex for $r>2$ and Schur-concave for $r<2$. This was also obtained in [1] as a consequence of Theorem A1.

Theorem 2.3. Let $f$ be a real continuous strictly monotone function on $I \subset \mathbb{R}$ and $g$ be a differentiable and strictly increasing function on $I$. Then, for the generalized weighted integral quasiarithmetic mean defined by

$$
M_{f}\left(g^{\prime} ; i d ; x, y\right)=f^{-1}\left(\frac{1}{\int_{x}^{y} g^{\prime}(t) d t} \int_{x}^{y} g^{\prime}(t) f(t) d t\right), \quad(x, y) \in I^{2},
$$

the following hold:

(i) $M_{f}\left(g^{\prime} ; i d ; x, y\right)$ is Schur-convex on $I^{2}$

if $f$ is increasing, and $g$ and $f \circ g^{-1}$ are convex

or if $f$ is decreasing and $g$ is convex and $f \circ g^{-1}$ is concave,

(ii) $M_{f}\left(g^{\prime} ;\right.$ id; $\left.x, y\right)$ is Schur-concave on $I^{2}$

if $f$ is decreasing and $g$ is concave and $f \circ g^{-1}$ is convex or

if $f$ is increasing, and $g$ and $f \circ g^{-1}$ are concave. 
Proof. Applying Theorem A1 and Lemma A3 (for $p \equiv 1$ ) for function $f \circ g^{-1}$, we conclude that

$$
\Phi(x, y)=\frac{1}{y-x} \int_{x}^{y}\left(f \circ g^{-1}\right)(u) d u
$$

is increasing (decreasing) and Schur-convex (Schur-concave) on $I^{2}$ if $f \circ g^{-1}$ is increasing (decreasing) and convex (concave) on $I$.

Using Lemma A2, we now deduce that

$$
\Psi(x, y)=\Phi(g(x), g(y))=\frac{1}{g(y)-g(x)} \int_{g(x)}^{g(y)}\left(f \circ g^{-1}\right)(u) d u
$$

is

(a) Schur-convex if $g$ is convex and $f \circ g^{-1}$ is convex and $f$ is increasing or if $g$ is concave and $f \circ g^{-1}$ is convex and $f$ is decreasing,

(b) Schur-concave if $g$ is concave and $f \circ g^{-1}$ is concave and $f$ is increasing or if $g$ is convex and $f \circ g^{-1}$ is concave and $f$ is decreasing.

Using substitution $u=g(t)$, we can rewrite

$$
\Psi(x, y)=\frac{1}{g(y)-g(x)} \int_{g(x)}^{g(y)}\left(f \circ g^{-1}\right)(u) d u=\frac{1}{\int_{x}^{y} g^{\prime}(t) d t} \int_{x}^{y} g^{\prime}(t) f(t) d t
$$

Finally, we apply Lemma A3 to $M_{f}\left(g^{\prime} ; i d ; x, y\right)=f^{-1}(\Psi(x, y))$ in order to conclude that $M_{f}\left(g^{\prime} ; i d ; x, y\right)$ is

(a') Schur-convex if $\Psi(x, y)$ is Schur-convex and $f^{-1}$ is increasing or if $\Psi(x, y)$ is Schurconcave and $f^{-1}$ is decreasing,

(b') Schur-concave if $\Psi(x, y)$ is Schur-convex and $f^{-1}$ is decreasing or if $\Psi(x, y)$ is Schurconcave and $f^{-1}$ is increasing.

Combining (a), (b), (a'), and (b') completes the proof.

In [8], a new symmetric mean was defined for two strictly monotone functions $f$ and $g$ on $I \subseteq \mathbb{R}$ as

$$
N(f, g ; x, y)=f^{-1}\left(\int_{0}^{1}\left(f \circ g^{-1}\right)(s g(x)+(1-s) g(y)) d s\right), \quad(x, y) \in I^{2} .
$$

If we change the variable $u=s g(x)+(1-s) g(y)$, we have

$$
N(f, g ; x, y)=f^{-1}\left(\frac{1}{g(y)-g(x)} \int_{g(x)}^{g(y)}\left(f \circ g^{-1}\right)(u) d u\right)
$$


Further, by substitution $u=g(t)$, we obtain

$$
N(f, g ; x, y)=f^{-1}\left(\frac{1}{\int_{x}^{y} g^{\prime}(t) d t} \int_{x}^{y} f(t) g^{\prime}(t) d t\right) .
$$

Note that under an additional assumption that $g$ is strictly increasing, we have $N(f, g ; x, y)=M_{f}\left(g^{\prime} ; i d ; x, y\right)$. Thus, using the same idea as in the proof of Theorem 2.3, an analogous result can easily be given for the mean $N(f, g ; x, y)$.

Theorem 2.4. Let $f$ and $g$ be real continuous strictly monotone functions on $I \subset \mathbb{R}$. Then, for the mean defined in (2.13), the following hold:

(i) $N(f, g ; x, y)$ is Schur-convex on $I^{2}$

if $f$ is increasing and $g$ is increasing and convex and $f \circ g^{-1}$ is convex

or if $f$ is increasing and $g$ is decreasing and concave and $f \circ g^{-1}$ is convex

or if $f$ is decreasing and $g$ is decreasing and concave and $f \circ g^{-1}$ is concave

or if $f$ is decreasing and $g$ is increasing and convex and $f \circ g^{-1}$ is concave,

(ii) $N(f, g ; x, y)$ is Schur-concave on $I^{2}$

if $f$ is decreasing and $g$ is decreasing and convex and $f \circ g^{-1}$ is convex

or if $f$ is decreasing and $g$ is increasing and concave and $f \circ g^{-1}$ is convex

or if $f$ is increasing and $g$ is increasing and concave and $f \circ g^{-1}$ is concave

or if $f$ is increasing and $g$ is decreasing and convex and $f \circ g^{-1}$ is concave.

\subsection{Application of Theorem A1 for the Extended Mean Values}

For $x, y>0$ and $r, s \in \mathbb{R}$, extended mean values were defined in [9] by Stolarsky as follows:

$$
E(r, s ; x, y)= \begin{cases}\left(\frac{r}{s} \cdot \frac{y^{s}-x^{s}}{y^{r}-x^{r}}\right)^{1 /(s-r)}, & r s(r-s)(x-y) \neq 0, \\ \left(\frac{1}{r} \cdot \frac{y^{r}-x^{r}}{\log y-\log x}\right)^{1 / r}, & s=0, r(x-y) \neq 0, \\ \frac{1}{e^{1 / r}}\left(\frac{x^{x^{r}}}{y^{y^{r}}}\right)^{1 /\left(x^{r}-y^{r}\right)}, & s=r, r(x-y) \neq 0, \\ \sqrt{x y}, & s=r=0, x \neq y, \\ x, & x=y .\end{cases}
$$

As a special case, the identric mean $I_{r}$ of order $r$ and the logarithmic mean $L_{r}$ of order $r$ are recaptured. Namely, $I_{r}(x, y)=E(r, r ; x, y)$ and $L_{r}(x, y)=E(r, 1 ; x, y)$.

On the other hand, note that the generalized weighted quasiarithmetic mean defined in (2.1) is a generalization of the extended means. Namely, $E(r, s ; a, b)=M_{f}\left(x^{r-1}, i d ; a, b\right)$ for $f(t)=t^{s-r}$.

Many properties of extended mean values have been considered in [10]. It was shown that $E(r, s ; x, y)$ are continuous on $\{(r, s ; x, y): r, s \in \mathbb{R}, x, y>0\}$ and symmetric with respect to both $r$ and $s$, and $x$ and $y$. 
Schur-convexity of the extended mean values $E(r, s ; x, y)$ with respect to $(r, s)$ and $(x, y)$ was considered in $[4,5,11]$.

Sándor in [12] (and also Qi et al. in [11]) proved the Schur-convexity of the extended mean values $E(r, s ; x, y)$ with respect to $(r, s)$, using Theorem A1 and the integral representation $\ln E(r, s ; x, y)=(1 /(s-r)) \int_{r}^{s} \ln I_{t}(x, y) d t$.

Shi et al. in [5], using Theorem A1 and Lemma A3 obtained the following condition for the Schur-convexity of the extended mean values $E(r, s ; x, y)$ with respect to $(x, y)$.

Theorem A4. For fixed $(r, s)$,

(i) if $2<2 r<s$ or $2 \leq 2 s \leq r$, then the extended mean values $E(r, s ; x, y)$ are Schur-convex with respect to $(x, y) \in(0,+\infty) \times(0,+\infty)$,

(ii) if $(r, s) \in\{r<s \leq 2 r, 0<r \leq 1\} \cup\{s<r \leq 2 s, 0<s \leq 1\} \cup\{0<s<r \leq 1\} \cup\{0<$ $r<s \leq 1\} \cup\{s \leq 2 r<0\} \cup\{r \leq 2 s<0\}$, then the extended mean values $E(r, s ; x, y)$ are Schur-concave with respect to $(x, y) \in(0,+\infty) \times(0,+\infty)$.

Remark 2.5. As a special case for $f(t)=t^{s-r}$ and $g(t)=t^{r}$ in Theorem 2.3, we recapture the result from Theorem A4 for the extended mean values

$$
\begin{aligned}
E(r, s ; x, y) & =M_{f}\left(g^{\prime} ; i d ; x, y\right)=\left(\frac{1}{\int_{x}^{y} r t^{r-1} d t} \int_{x}^{y} r t^{r-1} t^{s-r} d t\right)^{1 /(s-r)} \\
& =\left(\frac{1}{y^{r}-x^{r}} \int_{x^{r}}^{y^{r}}\left(t^{1 / r}\right)^{s-r} d t\right)^{1 /(s-r)} .
\end{aligned}
$$

Chu and Zhang in [13] established the necessary and sufficient conditions for the extended mean values $E(r, s ; x, y)$ to be Schur-convex (Schur-concave) with respect to $(x, y)$, for fixed $(r, s)$.

Theorem A5. For fixed $(r, s) \in \mathbb{R}^{2}$,

(i) the extended mean values $E(r, s ; x, y)$ are Schur-convex with respect to $(x, y) \in(0, \infty) \times$ $(0, \infty)$ if and only if $(r, s) \in\{s \geq 1, r \geq 1, s+r \geq 3\}$,

(ii) the extended mean values $E(r, s ; x, y)$ are Schur-concave with respect to $(x, y) \in(0, \infty) \times$ $(0, \infty)$ if and only if $(r, s) \in\{r \leq 1, s+r \leq 3\} \cup\{s \leq 1, s+r \leq 3\}$.

We remark that the above result does not cover the case $r=s$, that is, the case of the identric mean $I_{r}(x, y)$ of order $r$. Monotonicity and Schur-concavity of the identric mean $I_{r}(x, y)$ with respect to $(x, y)$ and for fixed $r$ was discussed in [14], using the hyperbolic composite function.

Theorem A6. For fixed $r \in \mathbb{R}$,

(i) $I_{r}(x, y)$ is increasing with respect to $(x, y) \in(0, \infty) \times(0, \infty)$,

(ii) if $0<r \leq 1$, then $I_{r}(x, y)$ is Schur-concave with respect to $(x, y) \in(0, \infty) \times(0, \infty)$.

\section{Convexity}

The following result is an extension of Wulbert's result from [2]. 
Theorem 3.1. Let $f$ be a continuous function on an interval I with a nonempty interior. If $f$ is convex on $I$, then the integral arithmetic mean $F$ defined in (1.3) is convex on $I^{2}$.

Furthermore, for $x_{i}, y_{i} \in I, i=1, \ldots, n$ and nonnegative real weights $w_{i}, i=1, \ldots, n$ such that $\sum_{i=1}^{n} w_{i}=1$, the following hold:

$$
\begin{gathered}
\frac{1}{\bar{y}-\bar{x}} \int_{\bar{x}}^{\bar{y}} f(t) d t \leq \sum_{i=1}^{n} w_{i} \frac{1}{y_{i}-x_{i}} \int_{x_{i}}^{y_{i}} f(t) d t \\
f\left(\frac{\bar{x}+\bar{y}}{2}\right) \leq \frac{1}{\bar{y}-\bar{x}} \int_{\bar{x}}^{\bar{y}} f(t) d t \leq \sum_{i=1}^{n} w_{i} \frac{1}{y_{i}-x_{i}} \int_{x_{i}}^{y_{i}} f(t) d t \leq \sum_{i=1}^{n} w_{i} \frac{f\left(x_{i}\right)+f\left(y_{i}\right)}{2},
\end{gathered}
$$

where $\bar{x}=\sum_{i=1}^{n} w_{i} x_{i}$ and $\bar{y}=\sum_{i=1}^{n} w_{i} y_{i}$.

Proof. Using the discrete Jensen inequality for the convex function $f$, we have the following conclusion:

$$
\begin{aligned}
\sum_{i=1}^{n} w_{i} F\left(x_{i}, y_{i}\right) & =\sum_{i=1}^{n} w_{i} \int_{0}^{1} f\left(y_{i} s+x_{i}(1-s)\right) d s \\
& =\int_{0}^{1} \sum_{i=1}^{n} w_{i} f\left(y_{i} s+x_{i}(1-s)\right) d s \\
& \geq \int_{0}^{1} f\left(\sum_{i=1}^{n} w_{i}\left(y_{i} s+x_{i}(1-s)\right)\right) d s \\
& =\int_{0}^{1} f\left(s \sum_{i=1}^{n} w_{i} y_{i}+(1-s) \sum_{i=1}^{n} w_{i} x_{i}\right) \\
& =F(\bar{x}, \bar{y}) .
\end{aligned}
$$

So, function $F$ is convex on $I^{2}$.

Using the Hermite-Hadamard inequality for the convex function $f$, we can extend inequality (3.1) on the left and on the right hand side as follows:

$$
f\left(\frac{\bar{x}+\bar{y}}{2}\right) \leq \frac{1}{\bar{y}-\bar{x}} \int_{\bar{x}}^{\bar{y}} f(t) d t \leq \sum_{i=1}^{n} w_{i} \frac{1}{y_{i}-x_{i}} \int_{x_{i}}^{y_{i}} f(t) d t \leq \sum_{i=1}^{n} w_{i} \frac{f\left(x_{i}\right)+f\left(y_{i}\right)}{2} .
$$

Corollary 3.2. Generalized logarithmic mean $L_{r}(x, y)$ defined by (2.16) is convex for $r>2$ and concave for $r<2$.

Proof. Apply Theorem 3.1 for $f(t)=t^{r-1}$. 
Remark 3.3. Theorem 3.1 is a generalization of the discrete Jensen inequality. For $x_{i}=y_{i}, i=$ $1, \ldots, n$, the inequality

$$
\sum_{i=1}^{n} w_{i} F\left(x_{i}, y_{i}\right) \geq F(\bar{x}, \bar{y})
$$

recaptures the Jensen inequality

$$
\sum_{i=1}^{n} w_{i} f\left(x_{i}\right) \geq f\left(\sum_{i=1}^{n} w_{i} x_{i}\right)
$$

Remark 3.4. The inequality (3.1) is strict if $f$ is a strictly convex function unless $x_{1}=x_{2}=\cdots=$ $x_{n}=y_{1}=y_{2}=\cdots=y_{n}$.

\subsection{Applications}

We recall the following definitions and remarks (see, e.g., [15]).

Definition 3.5. A function $f:(a, b) \rightarrow \mathbb{R}$ is exponentially convex if it is continuous and

$$
\sum_{i, j=1}^{n} \xi_{i} \xi_{j} f\left(x_{i}+x_{j}\right) \geq 0
$$

for every $n \in N$ and every $\xi_{i} \in \mathbb{R}, i=1, \ldots, n$ such that $x_{i}+x_{j} \in(a, b), 1 \leq i, j \leq n$.

Definition 3.6. A function $f: I \rightarrow \mathbb{R}^{+}$, where $I$ is an interval in $\mathbb{R}$, is said to be log convex if $\log f$ is convex, or equivalently, if for all $x, y \in I$ and all $\alpha \in[0,1]$, we have

$$
f(\alpha x+(1-\alpha) y) \leq f^{\alpha}(x) f^{1-\alpha}(y) .
$$

Remark 3.7. If $f:(a, b) \rightarrow \mathbb{R}^{+}$is exponentially convex, then $f$ is a log-convex function.

Consider a family of functions $\phi_{r}: \mathbb{R}^{+} \rightarrow \mathbb{R}, r \in \mathbb{R}$ from [15], defined as

$$
\phi_{r}(t)= \begin{cases}\frac{t^{r}}{r(r-1)}, & r \neq 0,1 \\ -\log t, & r=0 \\ t \log t, & r=1 .\end{cases}
$$

Now, we will give some applications of (3.1). 
Journal of Inequalities and Applications

Theorem 3.8. Let $\bar{x}=\sum_{i=1}^{n} w_{i} x_{i}, \bar{y}=\sum_{i=1}^{n} w_{i} y_{i}$, let $w_{i}, i=1, \ldots, n$ be nonnegative real weights such that $\sum_{i=1}^{n} w_{i}=1$ and $x_{i}, y_{i} \in I$. Let us define function

$$
T(r)=\sum_{i=1}^{n} w_{i} \frac{1}{y_{i}-x_{i}} \int_{x_{i}}^{y_{i}} \phi_{r}(t) d t-\frac{1}{\bar{y}-\bar{x}} \int_{\bar{x}}^{\bar{y}} \phi_{r}(t) d t,
$$

where $\phi_{r}$ is given by (3.9). Then, the following hold:

(i) the function $r \mapsto T(r)$ is continuous on $\mathbb{R}$,

(ii) for each $n \in \mathbb{N}$ and $r_{1}, \ldots, r_{n} \in \mathbb{R}$ matrix $\left[T\left(\left(r_{i}+r_{j}\right) / 2\right)\right]_{i, j=1}^{n}$ is positive semidefinite. Particularly,

$$
\operatorname{det}\left[T\left(\frac{r_{i}+r_{j}}{2}\right)\right]_{i, j=1}^{n} \geq 0
$$

(iii) the function $r \mapsto T(r)$ is exponentially convex on $\mathbb{R}$,

(iv) if $T(r)>0$, the function $r \mapsto T(r)$ is log-convex on $\mathbb{R}$,

(v) for $r_{i}, i=1,2,3$ such that $r_{1}<r_{2}<r_{3}$, one has

$$
\left(T\left(r_{2}\right)\right)^{r_{3}-r_{1}} \leq\left(T\left(r_{1}\right)\right)^{r_{3}-r_{2}}\left(T\left(r_{3}\right)\right)^{r_{2}-r_{1}} .
$$

Proof. Analogous to the proof of Theorem 2.2 from [15].

Following the steps of the proofs of Theorems 2.4 and 2.5 given in [15], we can prove the following two mean value theorems.

Theorem 3.9. Let $I$ be any compact interval, $\bar{x}=\sum_{i=1}^{n} w_{i} x_{i}, \bar{y}=\sum_{i=1}^{n} w_{i} y_{i}$, where $w_{i}, i=1, \ldots, n$ are nonnegative real weights such that $\sum_{i=1}^{n} w_{i}=1$ and $x_{i}, y_{i} \in I$. If $f \in C^{2}(I)$, then there exists $\xi \in I$ such that

$$
\frac{f^{\prime \prime}(\zeta)}{6}\left[\sum_{i=1}^{n} w_{i}\left(x_{i}^{2}+x_{i} y_{i}+y_{i}^{2}\right)-\left(\bar{y}^{2}+\overline{x y}+\bar{x}^{2}\right)\right]=\sum_{i=1}^{n} w_{i} \frac{1}{y_{i}-x_{i}} \int_{x_{i}}^{y_{i}} f(t) d t-\frac{1}{\bar{y}-\bar{x}} \int_{\bar{x}}^{\bar{y}} f(t) d t .
$$

Theorem 3.10. Let I be any compact interval and $\bar{x}, \bar{y}$ as in Theorem 3.9. If $f_{1}, f_{2} \in C^{2}(I)$ such that $f_{2}^{\prime \prime}(t)$ does not vanish for any value of $t \in I$, then there exists $\xi \in I$ such that

$$
\frac{f_{1}^{\prime \prime}(\xi)}{f_{2}^{\prime \prime}(\xi)}=\frac{\sum_{i=1}^{n} w_{i}\left(1 /\left(y_{i}-x_{i}\right)\right) \int_{x_{i}}^{y_{i}} f_{1}(t) d t-(1 /(\bar{y}-\bar{x})) \int_{\bar{x}}^{\bar{y}} f_{1}(t) d t}{\sum_{i=1}^{n} w_{i}\left(1 /\left(y_{i}-x_{i}\right)\right) \int_{x_{i}}^{y_{i}} f_{2}(t) d t-(1 /(\bar{y}-\bar{x})) \int_{\bar{x}}^{\bar{y}} f_{2}(t) d t}
$$

provided that denominator on right-hand side is nonzero. 
Remark 3.11. Let $\bar{x}=\sum_{i=1}^{n} w_{i} x_{i}, \bar{y}=\sum_{i=1}^{n} w_{i} y_{i}$, where $w_{i}, i=1, \ldots, n$ are nonnegative real weights such that $\sum_{i=1}^{n} w_{i}=1$ and $x_{i}, y_{i}$ in I. If the inverse of $f_{1}^{\prime \prime} / f_{2}^{\prime \prime}$ exists, then various kinds of means can be defined by (3.14). Namely,

$$
\xi=\left(\frac{f_{1}^{\prime \prime}}{f_{2}^{\prime \prime}}\right)^{-1}\left(\frac{\sum_{i=1}^{n} w_{i}\left(1 /\left(y_{i}-x_{i}\right)\right) \int_{x_{i}}^{y_{i}} f_{1}(t) d t-(1 /(\bar{y}-\bar{x})) \int_{\bar{x}}^{\bar{y}} f_{1}(t) d t}{\sum_{i=1}^{n} w_{i}\left(1 /\left(y_{i}-x_{i}\right)\right) \int_{x_{i}}^{y_{i}} f_{2}(t) d t-(1 /(\bar{y}-\bar{x})) \int_{\bar{x}}^{\bar{y}} f_{2}(t) d t}\right) .
$$

Moreover, we can define three-parameter means as in [15]

$$
M_{r, p}^{s}\left(w_{i} ; x_{i}^{s}, y_{i}^{s} ; n\right)=\left(\frac{T_{\mathrm{s}}(r)}{T_{\mathrm{s}}(p)}\right)^{1 /(r-p)}
$$

where, including all the limit cases,

$$
\begin{aligned}
& T_{s}(r)=\frac{s^{3}}{r^{3}-r s^{2}}\left[\sum_{i=1}^{n} w_{i} \frac{x_{i}^{r+s}-y_{i}^{r+s}}{x_{i}^{s}-y_{i}^{s}}-\frac{\left(M_{w_{i}}^{s}\left(x_{i}\right)\right)^{r+s}-\left(M_{w_{i}}^{s}\left(y_{i}\right)\right)^{r+s}}{\left(M_{w_{i}}^{s}\left(x_{i}\right)\right)^{s}-\left(M_{w_{i}}^{s}\left(y_{i}\right)\right)^{s}}\right], \quad r^{3}-r s^{2} \neq 0, s \neq 0 ; \\
& T_{s}(-s)=\log \left(\left(M_{w_{i}}^{s}\left(x_{i}\right)\right)^{s}-\left(M_{w_{i}}^{s}\left(y_{i}\right)\right)^{s} \sqrt{\frac{M_{w_{i}}^{s}\left(x_{i}\right)}{M_{w_{i}}^{s}\left(y_{i}\right)}} \prod_{i=1}^{n}\left(\frac{x_{i}}{y_{i}}\right)^{w_{i} /\left(x_{i}^{s}-y_{i}^{s}\right)}\right)^{s / 2}, \quad s \neq 0 ;
\end{aligned}
$$

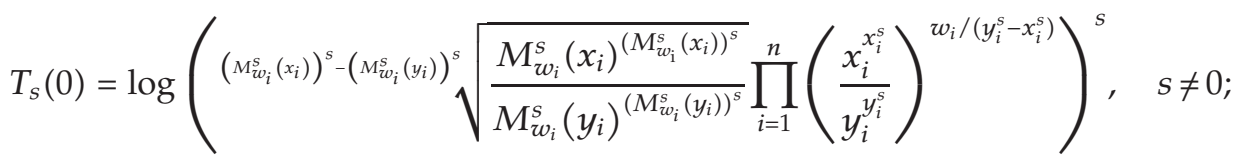

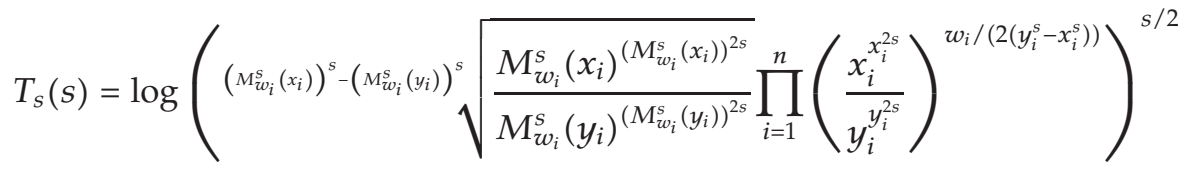

$$
\begin{aligned}
& -\frac{\left(M_{w}^{s}\left(x_{i}\right)\right)^{2 s}-\left(M_{w}^{s}\left(y_{i}\right)\right)^{2 s}}{4}+\sum_{i=1}^{n} w_{i} \frac{x_{i}^{2 s}-y_{i}^{2 s}}{4}, \quad s \neq 0, \\
& T_{0}(r)=\frac{1}{r^{3}}\left[\sum_{i=1}^{n} w_{i} \frac{x_{i}^{r}-y_{i}^{r}}{\log x_{i}-\log y_{i}}-\frac{\left(M_{w}^{0}\left(x_{i}\right)\right)^{r}-\left(M_{w}^{0}\left(y_{i}\right)\right)^{r}}{\log \left(M_{w}^{0}\left(x_{i}\right)\right)-\log \left(M_{w}^{0}\left(y_{i}\right)\right)}\right], \quad r \neq 0, \\
& T_{0}(0)=\frac{1}{6}\left[\sum_{i=1}^{n} w_{i} \frac{\log ^{3} x_{i}-\log ^{3} y_{i}}{\log x_{i}-\log y_{i}}-\frac{\left(\log M_{w}^{0}\left(x_{i}\right)\right)^{3}-\left(\log M_{w}^{0}\left(y_{i}\right)\right)^{3}}{\log \left(M_{w}^{0}\left(x_{i}\right)\right)-\log \left(M_{w}^{0}\left(y_{i}\right)\right)}\right]
\end{aligned}
$$

and the weighted power mean of $x_{i}$ is denoted as

$$
M_{w_{i}}^{s}\left(x_{i}\right)= \begin{cases}\left(\sum_{i=1}^{n} w_{i} x_{i}^{s}\right)^{1 / s}, & s \neq 0, \\ \prod_{i=1}^{n} x_{i}^{w_{i}}, & s=0 .\end{cases}
$$


All the limiting cases of (3.16) are given as follows:

$M_{r, r}^{s}\left(w_{i} ; x_{i}^{s}, y_{i}^{s} ; n\right)$

$$
=\exp \left(\frac{\frac{\left(M_{w_{i}}^{s}\left(x_{i}\right)\right)^{r+s} \log \left(M_{w_{i}}^{s}\left(x_{i}\right)\right)-\left(M_{w_{i}}^{s}\left(y_{i}\right)\right)^{r+s} \log \left(M_{w_{i}}^{s}\left(y_{i}\right)\right)}{\left(M_{w_{i}}^{s}\left(x_{i}\right)\right)^{s}-\left(M_{w_{i}}^{s}\left(y_{i}\right)\right)^{s}}}{r^{2}-r s}+\frac{-\sum_{i=1}^{n} w_{i} \frac{y_{i}^{r+s} \log y_{i}^{s}-x_{i}^{r+s} \log x_{i}^{s}}{y_{i}^{s}-x_{i}^{s}}}{\frac{\left(M_{w_{i}}^{s}\left(x_{i}\right)\right)^{r+s}-\left(M_{w_{i}}^{s}\left(y_{i}\right)\right)^{r+s}}{\left(M_{w_{i}}^{s}\left(x_{i}\right)\right)^{s}-\left(M_{w_{i}}^{s}\left(y_{i}\right)\right)^{s}}-\sum_{i=1}^{n} w_{i} \frac{y_{i}^{r+s}-x_{i}^{r+s}}{y_{i}^{s}-x_{i}^{s}}}\right),
$$

$M_{-s,-s}^{s}\left(w_{i} ; x_{i}^{s}, y_{i}^{s} ; n\right)$

$$
=\exp \left(\frac{3}{2 s}+\frac{\frac{\log ^{2}\left(M_{w_{i}}^{s}\left(x_{i}\right)\right)-\log ^{2}\left(M_{w_{i}}^{s}\left(y_{i}\right)\right)}{\left(M_{w_{i}}^{s}\left(x_{i}\right)\right)^{s}-\left(M_{w_{i}}^{s}\left(y_{i}\right)\right)^{s}}-\sum_{i=1}^{n} w_{i} \frac{\log ^{2} x_{i}^{s}-\log ^{2} y_{i}^{s}}{x_{i}^{s}-y_{i}^{s}}}{\frac{\log \left(M_{w_{i}}^{s}\left(x_{i}\right)\right)-\log \left(M_{w_{i}}^{s}\left(y_{i}\right)\right)}{\left(M_{w_{i}}^{s}\left(x_{i}\right)\right)^{s}-\left(M_{w_{i}}^{s}\left(y_{i}\right)\right)^{S}}-\sum_{i=1}^{n} w_{i} \frac{\log x_{i}^{s}-\log y_{i}^{s}}{x_{i}^{s}-y_{i}^{S}}}\right),
$$

$M_{0,0}^{s}\left(w_{i} ; x_{i}^{s}, y_{i}^{s} ; n\right)$

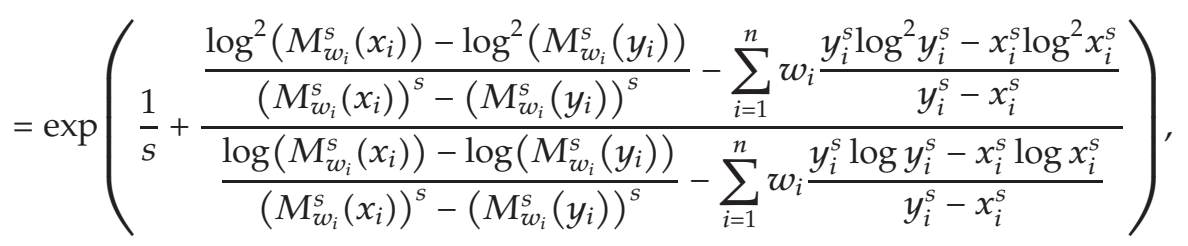

$M_{s, s}^{s}\left(w_{i} ; x_{i}^{s}, y_{i}^{s} ; n\right)$

$$
\exp \left(\begin{array}{c}
\frac{\left(M_{w_{i}}^{s}\left(x_{i}\right)\right)^{2 s} \log ^{2}\left(M_{w_{i}}^{s}\left(x_{i}\right)\right)-\left(M_{w_{i}}^{s}\left(y_{i}\right)\right)^{2 s} \log ^{2}\left(M_{w_{i}}^{s}\left(y_{i}\right)\right)}{\left(M_{w_{i}}^{s}\left(x_{i}\right)\right)^{s}-\left(M_{w_{i}}^{s}\left(y_{i}\right)\right)^{s}} \\
-\frac{1}{s}+\frac{-\sum_{i=1}^{n} w_{i} \frac{y_{i}^{2 s} \log ^{2} y_{i}^{s}-x_{i}^{2 s} \log ^{2} x_{i}^{s}}{y_{i}^{s}-x_{i}^{s}}}{\frac{\left(M_{w_{i}}^{s}\left(x_{i}\right)\right)^{2 s} \log \left(M_{w_{i}}^{s}\left(x_{i}\right)\right)-\left(M_{w_{i}}^{s}\left(y_{i}\right)\right)^{2 s} \log \left(M_{w_{i}}^{s}\left(y_{i}\right)\right)}{\left(M_{w_{i}}^{s}\left(x_{i}\right)\right)^{s}-\left(M_{w_{i}}^{s}\left(y_{i}\right)\right)^{s}}} \\
-\sum_{i=1}^{n} w_{i} \frac{y_{i}^{2 s} \log y_{i}^{s}-x_{i}^{2 s} \log x_{i}^{s}}{y_{i}^{s}-x_{i}^{s}}
\end{array}\right),
$$




$$
\begin{aligned}
& M_{r, r}^{0}\left(w_{i} ; \log \left(x_{i}\right), \log \left(y_{i}\right) ; n\right) \\
& =\exp \left(\begin{array}{c}
\frac{\left(M_{w_{i}}^{0}\left(x_{i}\right)\right)^{r} \log \left(M_{w_{i}}^{0}\left(x_{i}\right)\right)-\left(M_{w_{i}}^{0}\left(y_{i}\right)\right)^{r} \log \left(M_{w_{i}}^{0}\left(y_{i}\right)\right)}{\log \left(M_{w_{i}}^{0}\left(x_{i}\right)\right)-\log \left(M_{w_{i}}^{0}\left(y_{i}\right)\right)} \\
-\frac{2}{r}+\frac{-\sum_{i=1}^{n} w_{i} \frac{x_{i}^{r} \log x_{i}-y_{i}^{r} \log y_{i}}{\log x_{i}-\log y_{i}}}{\frac{\left(M_{w_{i}}^{0}\left(x_{i}\right)\right)^{r}-\left(M_{w_{i}}^{0}\left(y_{i}\right)\right)^{r}}{\log \left(M_{w_{i}}^{0}\left(x_{i}\right)\right)-\log \left(M_{w_{i}}^{0}\left(y_{i}\right)\right)}-\sum_{i=1}^{n} w_{i} \frac{x_{i}^{r}-y_{i}^{r}}{\log x_{i}-\log y_{i}}}
\end{array}\right), \\
& M_{0,0}^{0}\left(w_{i} ; \log \left(x_{i}\right), \log \left(y_{i}\right) ; n\right) \\
& =\exp \left(\frac{1}{3} \frac{\frac{\log ^{3}\left(M_{w_{i}}^{0}\left(x_{i}\right)\right)-\log ^{3}\left(M_{w_{i}}^{0}\left(y_{i}\right)\right)}{\log \left(M_{w_{i}}^{0}\left(x_{i}\right)\right)-\log \left(M_{w_{i}}^{0}\left(y_{i}\right)\right)}-\sum_{i=1}^{n} w_{i} \frac{\left(\log x_{i}\right)^{3}-\left(\log y_{i}\right)^{3}}{\log x_{i}-\log y_{i}}}{\log \left(M_{w_{i}}^{0}\left(x_{i}\right)\right)+\log \left(M_{w_{i}}^{0}\left(y_{i}\right)\right)-\sum_{i=1}^{n} w_{i} \frac{\left(\log x_{i}\right)^{2}-\left(\log y_{i}\right)^{2}}{\log x_{i}-\log y_{i}}}\right) .
\end{aligned}
$$

Theorem 3.12. Let $\bar{x}=\sum_{i=1}^{n} w_{i} x_{i}, \bar{y}=\sum_{i=1}^{n} w_{i} y_{i}$, where $w_{i}, i=1, \ldots, n$ are nonnegative real weights such that $\sum_{i=1}^{n} w_{i}=1$ and $x_{i}, y_{i} \in I$. If $r, p, u, v \in \mathbb{R}$ are such that $r \leq u, p \leq v$, then the following inequality is valid:

$$
M_{r, p}^{s}\left(w_{i} ; x_{i}, y_{i} ; n\right) \leq M_{u, v}^{s}\left(w_{i} ; x_{i}, y_{i} ; n\right)
$$

Proof. It follows the steps of the proof of Theorem 4.2 given in [15].

Remark 3.13. As a special case for $x_{i}=y_{i}, i=1, \ldots, n$, we recapture the discrete version of the results obtained in [16].

\section{Hermite-Hadamard Inequality}

Let us recall the Hermite-Hadamard inequality: if $f: I \rightarrow \mathbb{R}$ is a convex function on $I$ and $a, b \in I$ such that $a<b$, then the following double inequality holds:

$$
f\left(\frac{a+b}{2}\right) \leq \frac{1}{b-a} \int_{a}^{b} f(t) d t \leq \frac{f(a)+f(b)}{2} .
$$

In [17], it was shown that $f$ is convex if and only if at least one of the inequalities in (4.1) is valid.

An interesting fact is that the original proof of Theorem A1 was given using the second Hermite-Hadamard inequality and the first one follows from the same theorem. 
A very interesting inequality closely connected with the Hermite-Hadamard inequality was given in [18]. Namely, it was shown by a simple geometric argument that for a convex function $f$, the following is valid:

$$
0 \leq \frac{1}{b-a} \int_{a}^{b} f(t) d t-f\left(\frac{a+b}{2}\right) \leq \frac{f(a)+f(b)}{2}-\frac{1}{b-a} \int_{a}^{b} f(t) d t
$$

The same inequality was rediscovered later in [19] through an elementary analytic proof.

\subsection{Application of Theorem A1 for a Function Connected with Hadamard Inequality}

Dragomir et al. in [20] (see also [21, page 108]) considered a function $L:[0,1] \rightarrow \mathbb{R}$, connected to Hadamard's inequality, given by

$$
L(t)=\frac{1}{2(b-a)} \int_{a}^{b}[f(t a+(1-t) x)+f(t b+(1-t) x)] d x
$$

where $f: I \subseteq \mathbb{R} \rightarrow \mathbb{R}$ and $a, b \in I$ with $a<b$, and showed convexity of $L$ if $f$ is convex function on $I$.

Yang and Hong, in [22] (see also [21, page 147]) considered a similar function. Shi, in [23], found a similar result as Theorem A1 for the function $L$.

Theorem A7. Let $I \subseteq \mathbb{R}$ be an interval with a nonempty interior and $f$ be a continuous function on I. For function $P_{L}(a, b)$ defined on $I^{2}$ as

$$
P_{L}(a, b)= \begin{cases}L(t), & a, b \in I, a \neq b \\ f(a), & a=b\end{cases}
$$

the following hold:

(i) for $1 / 2 \leq t \leq 1$, if $f$ is convex on $I$, then $P_{L}$ is Schur-convex on $I^{2}$,

(ii) for $0 \leq t \leq 1 / 2$, if $f$ is concave on $I$, then $P_{L}$ is Schur-concave on $I^{2}$.

In [24], we obtained Schur-convexity of the Čebišev functional. In note [25], our first aim was to give another similar result to Theorem A1.

Theorem A8. Let $I \subseteq \mathbb{R}$ be an interval with a nonempty interior. Let $f$ be a continuous function on $I$ and $\alpha$ a continuous function on $[0,1]$. Let $L_{\alpha}:[0,1] \rightarrow \mathbb{R}$ be a function defined by

$$
L_{\alpha}(t)=\frac{1}{b-a} \int_{a}^{b}[f(\alpha(t) a+(1-\alpha(t)) x)+f(\alpha(t) b+(1-\alpha(t)) x)] d x .
$$


For a function $P_{\alpha}(a, b)$ defined on $I^{2}$ as

$$
P_{\alpha}(a, b)= \begin{cases}L_{\alpha}(t), & a, b \in I, a \neq b, \\ f(a), & a=b\end{cases}
$$

the following hold:

(i) for $\alpha$ such that $\min _{t \in I} \alpha(t)=1 / 2, \max _{t \in I} \alpha(t)=1$, if $f$ is convex on I, then $P_{\alpha}$ is Schurconvex on $I^{2}$,

(ii) for $\alpha$ such that $\min _{t \in I} \alpha(t)=0, \max _{t \in I} \alpha(t)=1 / 2$, if $f$ is concave on $I$, then $P_{\alpha}$ is Schurconcave on $I^{2}$.

Another function defined by a double integral in connection with the HermiteHadamard inequalities is considered in [26]

$$
G(t)=\frac{1}{(b-a)^{2}} \iint_{a}^{b} f(t x+(1-t) y) d x d y
$$

Shi, in [23], found a similar result as Theorem A1 for this function $G(t)$.

Theorem A9. Let $I \subseteq \mathbb{R}$ be an interval with a nonempty interior, $f$ a continuous function on $I$, and $0 \leq t \leq 1$. If $f$ is convex (concave) on $I$, the function $Q(a, b)$ defined on $I^{2}$ as

$$
Q(a, b)= \begin{cases}G(t), & a, b \in I, a \neq b \\ f(a), & a=b\end{cases}
$$

is Schur-convex (Schur-concave) on $I^{2}$.

\subsection{Schur-Convexity of Hermite-Hadamard Differences}

In [27], the property of Schur-convexity of the difference between the middle part and the left-hand side of the Hermite-Hadamard inequality (4.1), and the difference between the right-hand side and the middle part of the same inequality, was investigated. The following theorems were proved.

Theorem A10. Suppose $I$ is an open interval and $f: I \rightarrow \mathbb{R}$ is a continuous function. Function

$$
L(x, y)= \begin{cases}\frac{1}{y-x} \int_{x}^{y} f(t) d t-f\left(\frac{x+y}{2}\right), & x, y \in I, x \neq y \\ 0, & x=y \in I\end{cases}
$$

is Schur-convex (concave) on $I^{2}$ if and only if $f$ is convex (concave) on $I$. 
Journal of Inequalities and Applications

Theorem A11. Suppose $I$ is an open interval and $f: I \rightarrow \mathbb{R}$ is a continuous function. Function

$$
R(x, y)= \begin{cases}\frac{f(x)+f(y)}{2}-\frac{1}{y-x} \int_{x}^{y} f(t) d t, & x, y \in I, x \neq y \\ 0, & x=y \in I\end{cases}
$$

is Schur-convex (concave) on $I^{2}$ if and only if $f$ is convex (concave) on I.

First, we state a simple consequence of Theorems A1, A10, and A11.

Corollary 4.1. Let $f: I \subseteq \mathbb{R} \rightarrow \mathbb{R}$ be a continuous function. Then, the following statements are equivalent:

(i) $f$ is convex (concave),

(ii) $F$ is Schur-convex (Schur-concave),

(iii) L is Schur-convex (Schur-concave),

(iv) $R$ is Schur-convex (Schur-concave),

where $F$ is defined as in (1.4), $L$ as in (4.9) and $R$ as in (4.10).

Remark 4.2. It is not difficult to verify that

$$
\frac{\partial F}{\partial x}-\frac{\partial F}{\partial y}=\frac{\partial L}{\partial x}-\frac{\partial L}{\partial y}
$$

which, after applying Lemma A1, is another proof of (ii) $\Leftrightarrow($ iii) in Corollary 4.1.

In [28], the following identity was derived: if $f: I \subseteq \mathbb{R} \rightarrow \mathbb{R}$ is such that $f^{(n-1)}$ is absolutely continuous for some $n \geq 2, a, b \in I, a<b$ and $x \in[a, b]$, then

$$
\begin{aligned}
\frac{1}{b-a} \int_{a}^{b} f(t) d t= & f(x)+\sum_{i=0}^{n-2} f^{(i+1)}(x) \frac{(b-x)^{i+2}-(a-x)^{i+2}}{(b-a)(i+2) !} \\
& +\frac{1}{n !(b-a)}\left(\int_{a}^{x}(a-t)^{n} f^{(n)}(t) d t+\int_{x}^{b}(b-t)^{n} f^{(n)}(t) d t\right) .
\end{aligned}
$$

Applying identity (4.12) for $n=2$, then choosing, respectively, $x=a$ and $x=b$, adding up two thus obtained identities, and finally dividing by two procures

$$
\frac{1}{b-a} \int_{a}^{b} f(t) d t-\frac{f(a)+f(b)}{2}+\frac{b-a}{4}\left[f^{\prime}(b)-f^{\prime}(a)\right]=\frac{1}{4(b-a)} \int_{a}^{b}\left[(b-t)^{2}+(a-t)^{2}\right] f^{\prime \prime}(t) d t .
$$

Identity (4.13) enables us to give a new proof of sufficiency in Theorem A11. 
Proof of sufficiency in Theorem A11. We have

$$
(y-x)\left(\frac{\partial R}{\partial y}-\frac{\partial R}{\partial x}\right)=\frac{2}{y-x} \int_{x}^{y} f(t) d t-[f(y)+f(x)]+\frac{1}{2}(y-x)\left[f^{\prime}(y)-f^{\prime}(x)\right]
$$

Using (4.13), we see that in fact

$$
(y-x)\left(\frac{\partial R}{\partial y}-\frac{\partial R}{\partial x}\right)=\frac{1}{2(y-x)} \int_{x}^{y}\left[(y-t)^{2}+(x-t)^{2}\right] f^{\prime \prime}(t) d t
$$

Since by assumption $f$ is convex (concave), Lemma A1 yields that $R$ is Schur-convex (Schurconcave).

Remark 4.3. Note that with an additional assumption that $f \in C^{2}(I)$, since(27)is valid for all $x, y \in I$, from (4.15) necessity in Theorem A11 follows as well.

Identity similar to (4.13) can be found in [29]: if $f:[a, b] \rightarrow \mathbb{R}$ is twice differentiable, then the following identity is valid:

$$
\frac{1}{b-a} \int_{a}^{b} f(t) d t-\frac{f(a)+f(b)}{2}+\frac{b-a}{8}\left[f^{\prime}(b)-f^{\prime}(a)\right]=\frac{1}{2(b-a)} \int_{a}^{b}\left(t-\frac{a+b}{2}\right)^{2} f^{\prime \prime}(t) d t .
$$

With the help of identity (4.16), we can present the following.

Theorem 4.4. If $f: I \subseteq \mathbb{R} \rightarrow \mathbb{R}$ is a convex (concave) function, then the function

$$
P(x, y)= \begin{cases}\frac{f(x)+f(y)}{4}+\frac{1}{2} f\left(\frac{x+y}{2}\right)-\frac{1}{y-x} \int_{x}^{y} f(t) d t, & x, y \in I, x \neq y, \\ 0, & x=y \in I\end{cases}
$$

is Schur-convex (Schur-concave).

If $f \in C^{2}(I)$ and $P$ is Schur-convex (Schur-concave), then $f$ is convex (concave).

Proof. Using (4.16), we deduce

$$
\begin{aligned}
(y-x)\left(\frac{\partial P}{\partial y}-\frac{\partial P}{\partial x}\right) & =\frac{2}{y-x} \int_{x}^{y} f(t) d t-[f(y)+f(x)]+\frac{y-x}{4}\left[f^{\prime}(y)-f^{\prime}(x)\right] \\
& =\frac{1}{y-x} \int_{x}^{y}\left(t-\frac{x+y}{2}\right)^{2} f^{\prime \prime}(t) d t
\end{aligned}
$$

If $f$ is convex (concave), from Lemma $\mathrm{A} 1$, it follows that $P$ is Schur-convex (Schur-concave). 
Now, assume in addition that $f \in C^{2}(I)$. Applying the integral mean value theorem yields that there exists $\xi \in(x, y)$ such that

$$
(y-x)\left(\frac{\partial P}{\partial y}-\frac{\partial P}{\partial x}\right)=f^{\prime \prime}(\xi) \frac{1}{y-x} \int_{x}^{y}\left(t-\frac{x+y}{2}\right)^{2} d t=\frac{(y-x)^{2}}{12} f^{\prime \prime}(\xi)
$$

and this is valid for all $x, y \in I$. Since by assumption $P$ is Schur-convex (Schur-concave), from Lemma A1, it follows that $f$ is convex (concave).

Remark 4.5. If $P(x, y)$ is Schur-convex, since $((x+y) / 2,(x+y) / 2) \prec(x, y)$, one has

$$
\begin{aligned}
P\left(\frac{x+y}{2}, \frac{x+y}{2}\right) & \leq P(x, y) \\
& \Longleftrightarrow 0 \leq \frac{f(x)+f(y)}{4}+\frac{1}{2} f\left(\frac{x+y}{2}\right)-\frac{1}{y-x} \int_{x}^{y} f(t) d t \\
& \Longleftrightarrow \frac{1}{y-x} \int_{x}^{y} f(t) d t-f\left(\frac{x+y}{2}\right) \leq \frac{f(x)+f(y)}{2}-\frac{1}{y-x} \int_{x}^{y} f(t) d t
\end{aligned}
$$

which is exactly (4.2). Since in Theorem 4.4 we have shown that $P$ is Schur-convex if $f$ is convex, this is in fact a new proof of (4.2).

\section{Convexity and Schur-Convexity of Divided Differences}

In this final section, we turn our attention towards divided differences. Let us first recall the definition.

Definition 5.1. Let $f:[a, b] \rightarrow \mathbb{R}$. A $n$ th-order divided difference of $f$ at distinct points $x_{0}, \ldots, x_{n} \in[a, b]$ is defined recursively by

$$
\begin{gathered}
{\left[x_{i}\right] f=f\left(x_{i}\right), \quad i=0, \ldots, n,} \\
{\left[x_{0}, \ldots, x_{n}\right] f=\frac{\left[x_{1}, \ldots, x_{n}\right] f-\left[x_{0}, \ldots, x_{n-1}\right] f}{x_{n}-x_{0}} .}
\end{gathered}
$$

Notion closely related to divided differences is $n$-convexity.

Definition 5.2. A function $f:[a, b] \rightarrow \mathbb{R}$ is said to be $n$-convex on $[a, b], n \geq 0$, if and only if for all choices of $n+1$ distinct points in $[a, b]$,

$$
\left[x_{0}, \ldots, x_{n}\right] f \geq 0
$$

If the inequality is reversed, then $f$ is said to be $n$-concave on $[a, b]$.

For more details on divided differences and $n$-convexity, see [7]. 
In [30], Zwick proved the following theorem.

Theorem A12. Let $f$ be $(n+2)$-convex on $(a, b)$. Then, the function

$$
G(x)=\left[x, x+h_{1}, \ldots, x+h_{n}\right] f
$$

is a convex function of $x$ for all $x$ and all $h_{1}, \ldots, h_{n}$ such that $x+h_{i} \in(a, b), i=1, \ldots, n$.

Therefore, for $p_{i}>0$ and $x_{i} \in I, i=1, \ldots, m$, where $I$ is the domain of $G$, Jensen's inequality yields

$$
\frac{1}{P_{m}} \sum_{i=1}^{m} p_{i}\left[x_{i}, x_{i}+h_{1}, \ldots, x_{i}+h_{n}\right] f \geq\left[\bar{x}, \bar{x}+h_{1}, \ldots, \bar{x}+h_{n}\right] f,
$$

where $\bar{x}=1 / P_{m} \sum_{i=1}^{m} p_{i} x_{i}$.

This theorem is a generalization of a result from [31], where only 3-convex functions were considered. An additional generalization was given by Farwig and Zwick in [32].

Theorem A13. Let $f$ be $(n+2)$-convex on $(a, b)$. Then,

$$
G(\mathbf{x})=\left[x_{0}, \ldots, x_{n}\right] f
$$

is a convex function of the vector $\mathbf{x}=\left(x_{0}, \ldots, x_{n}\right)$. Consequently,

$$
\left[\sum_{i=0}^{m} a_{i} x_{0}^{i}, \ldots, \sum_{i=0}^{m} a_{i} x_{n}^{i}\right] f \leq \sum_{i=0}^{m} a_{i}\left[x_{0}^{i}, \ldots, x_{n}^{i}\right] f
$$

holds for all $a_{i} \geq 0$ such that $\sum_{i=0}^{m} a_{i}=1$, which is a generalization of (5.4).

Note that the divided difference is a permutation symmetric function. Thus, the following theorem follows from Theorem A13 and a result on majorization inequalities. It was obtained in [33] by Pečaric' and Zwick.

Theorem A14. Let $f$ be an $(n+2)$-convex function on $(a, b)$. If $(\mathbf{x}, \mathbf{y}) \in(a, b)^{n+1}$ and $\mathbf{x}>\mathbf{y}$, then

$$
\left[x_{0}, \ldots, x_{n}\right] f \geq\left[y_{0}, \ldots, y_{n}\right] f
$$

that is, function G defined in (5.5) is Schur-convex.

Many more results involving divided differences were obtained, among others the multivariate analogues, all of which can be found in [7].

About a decade later, Merkle in [34] presented the following. 
Theorem A15. Let $f$ be differentiable on $I \subseteq \mathbb{R}$ and $f^{\prime}$ continuous on I. Define

$$
D(x, y)= \begin{cases}\frac{f(y)-f(x)}{y-x}, & x, y \in I, x \neq y \\ f^{\prime}(x), & x=y \in I .\end{cases}
$$

Then, the conditions $(A)-(E)$ are equivalent and the conditions $(A)-\left(E^{\prime}\right)$ are equivalent, where
(A) $f^{\prime}$ is convex on $I$,
(B) $f^{\prime}((x+y) / 2) \leq D(x, y)$ for all $x, y \in I$,
(C) $D(x, y) \leq\left(f^{\prime}(x)+f^{\prime}(y)\right) / 2$ for all $x, y \in I$,
(D) $D$ is convex on $I^{2}$,
(E) $D$ is Schur-convex on $I^{2}$

and
(A') $f^{\prime}$ is concave on $I$,
(B') $f^{\prime}((x+y) / 2) \geq D(x, y)$ for all $x, y \in I$,
(C') $D(x, y) \geq\left(f^{\prime}(x)+f^{\prime}(y)\right) / 2$ for all $x, y \in I$,
(D') $D$ is concave on $I^{2}$,
(E') $D$ is Schur-concave on $I^{2}$.

First, note that function $D$ defined in (5.8) is the 1st-order divided difference of function $f$. Also,

$$
D(x, y)=\frac{1}{y-x} \int_{x}^{y} f^{\prime}(t) d t
$$

Thus, it becomes clear that the statements $(A) \Leftrightarrow(E)$ and $\left(A^{\prime}\right) \Leftrightarrow\left(E^{\prime}\right)$ are in fact an alternative statement of Theorem A1. Furthermore, implications $(A) \Rightarrow(E)$ and $\left(A^{\prime}\right) \Rightarrow\left(E^{\prime}\right)$ are a special case of Theorem $A 14$, while $(A) \Rightarrow(D)$ and $\left(A^{\prime}\right) \Rightarrow\left(D^{\prime}\right)$ are a special case of Theorem A13.

Moreover, note that $(B)$ and $(C)$, that is, $\left(B^{\prime}\right)$ and $\left(C^{\prime}\right)$, are in fact the HermiteHadamard inequalities and we have already commented on their relation with Theorem A1-one side is used in the proof and the other is a consequence of the theorem.

Implications $(D) \Rightarrow(E)$ and $\left(D^{\prime}\right) \Rightarrow\left(E^{\prime}\right)$ are trivial, since $D$ is symmetric.

Furthermore, the statements $(A) \Leftrightarrow(D)$ and $\left(A^{\prime}\right) \Leftrightarrow\left(D^{\prime}\right)$ are an alternative statement of Zhang and Chu's result from [3] and the necessity part recaptures Wulbert's result from [2] and the result from our Theorem 3.1. 


\subsection{Applications of Schur-Convexity of Divided Differences}

In [35], Yang introduced the following mean: let $f: \mathbb{R}^{+} \times \mathbb{R}^{+} \rightarrow \mathbb{R}^{+}$be a symmetric and positively homogeneous function (i.e., such that for $\lambda>0, f(\lambda x, \lambda y)=\lambda f(x, y))$, satisfying $f(1,1)=1$. For $p, q \in \mathbb{R}$, the two-parameter family generated by $f$ is defined as

$$
\mathscr{H}_{f}(p, q ; x, y)= \begin{cases}\left(\frac{f\left(x^{p}, y^{p}\right)}{f\left(x^{q}, y^{q}\right)}\right)^{1 /(p-q)}, & p \neq q, \\ \exp \left(\frac{d}{d p} \log f\left(x^{p}, y^{p}\right)\right), & p=q \neq 0, \\ \sqrt{x y}, & p=q=0 .\end{cases}
$$

Note that the extended mean vales $E(r, s ; x, y)$ and the Gini means

$$
G(r, s ; x, y)= \begin{cases}\left(\frac{x^{r}+y^{r}}{x^{s}+y^{s}}\right)^{1 /(r-s)}, & r \neq s, \\ \exp \left(\frac{x^{r} \log x+y^{r} \log y}{x^{r}+y^{r}}\right), & r=s\end{cases}
$$

are obtained as special cases of this new mean. In [36], necessary conditions under which Gini means (5.11) are Schur-convex and Schur-concave were given. In the short note [37], Witkowski completed this result with the proof of sufficiency of those conditions.

In a series of papers, Yang investigated various properties of the mean $\mathscr{\ell}_{f}$, such as monotonicity and logarithmic convexity. In [38], Witkowski continued his research by extending his results, giving simplified proofs and other conditions equivalent to monotonicity and convexity of $\mathscr{H}_{f}$. In order to do this, he introduced the function: $\widehat{f}(t)=$ $\log f(\exp (t), 1)$, so as to present $\mathscr{L}_{f}$ in the form

$$
\mathscr{H}_{f}(p, q ; x, y)=y \exp \frac{\widehat{f}(p \log (x / y))-\widehat{f}(q \log (x / y))}{p-q} .
$$

Using this form and Theorem A15, he proved the following.

Theorem A16. The following conditions are equivalent:

(a) for all $p, q \geq 0$ and all $x, y>0, \log \mathscr{\ell}_{f}$ is convex (concave) in $p$ and $q$,

(b) for all $p, q \geq 0$ and all $x, y>0, \log \mathscr{\ell}_{f}$ is Schur-convex (Schur-concave) in $p$ and $q$,

(c) $\widehat{f}^{\prime}(t)$ is convex (concave) for $t \geq 0$,

(d) for all $p, q \leq 0$ and all $x, y>0, \log \mathscr{L}_{f}$ is concave (convex) in $p$ and $q$,

(e) for all $p, q \leq 0$ and all $x, y>0, \log \mathscr{H}_{f}$ is Schur-concave (Schur-convex) in $p$ and $q$,

(f) $\hat{f}^{\prime}(t)$ is concave (convex) for $t \leq 0$. 
If $f$ is positively homogeneous, then so are $\mathscr{l}_{f}$ for every $(r, s)$ and so the fourparameter family can be created by

$$
\mathcal{F}_{f}(p, q ; r, s ; x, y)=\mathscr{\ell}_{\mathscr{d}_{f}(r, s)}(p, q ; x, y) .
$$

Since

$$
\mathscr{H}_{f} \widehat{(r, s)}(t)=\frac{\widehat{f}(r t)-\widehat{f}(s t)}{r-s},
$$

Witkowski was able to apply all the results obtained for the two-parameter means, in particular Theorem A16, for this new family of means. The one of special interest to us is the following.

Theorem A17. If $r+s>0$, the following conditions are equivalent:

(a) for all $p, q \geq 0$ and all $x, y>0, \log \Psi_{f}$ is convex (concave) in $p$ and $q$,

(b) for all $p, q \geq 0$ and all $x, y>0, \log \mathscr{F}_{f}$ is Schur-convex (Schur-concave) in $p$ and $q$,

(c) $t^{3} \widehat{f}^{\prime \prime \prime}(t)$ increases (decreases) for $t \geq 0$,

(d) for all $p, q \leq 0$ and all $x, y>0, \log \Psi_{f}$ is concave (convex) in $p$ and $q$,

(e) for all $p, q \leq 0$ and all $x, y>0, \log \Psi_{f}$ is Schur-concave (Schur-convex) in $p$ and $q$,

(f) $t^{3} \widehat{f}^{\prime \prime \prime}(t)$ decreases (increases) for $t \leq 0$.

If $r+s<0$, then the conditions $(c)$ and $(f)$ reverse.

Note that the same four-parameter family of means was the object of interest to Yang in [39]. He gave conditions under which $\mathcal{F}_{f}$ are increasing (decreasing) and logarithmically convex (logarithmically concave). Necessary and sufficient conditions for $\mathcal{F}_{f}$ to be increasing (decreasing) were, however, given in [38].

\section{Acknowledgments}

The research of the authors was supported by the Croatian Ministry of Science, Education and Sports, under the Research Grants nos. 117-1170889-0888 (for V. Čuljak and J. Pečarić) and 058-1170889-1050 (for I. Franjić).

\section{References}

[1] N. Elezović and J. Pečarić, "A note on Schur-convex functions," The Rocky Mountain Journal of Mathematics, vol. 30, no. 3, pp. 853-856, 2000.

[2] D. E. Wulbert, "Favard's inequality on average values of convex functions," Mathematical and Computer Modelling, vol. 37, no. 12-13, pp. 1383-1391, 2003.

[3] X. M. Zhang and Y. M. Chu, "Convexity of the integral arithmetic mean of a convex function," The Rocky Mountain Journal of Mathematics, vol. 40, no. 3, pp. 1061-1068, 2010.

[4] F. Qi, J. Sándor, S. S. Dragomir, and A. Sofo, "Notes on the Schur-convexity of the extended mean values," Taiwanese Journal of Mathematics, vol. 9, no. 3, pp. 411-420, 2005. 
[5] H.-N. Shi, S.-H. Wu, and F. Qi, "An alternative note on the Schur-convexity of the extended mean values," Mathematical Inequalities E Applications, vol. 9, no. 2, pp. 219-224, 2006.

[6] A. W. Marshall and I. Olkin, Inequalities: Theory of Majorization and Its Applications, vol. 143 of Mathematics in Science and Engineering, Academic Press, New York, NY, USA, 1979.

[7] J. E. Pečarić, F. Proschan, and Y. L. Tong, Convex Functions, Partial Orderings, and Statistical Applications, vol. 187 of Mathematics in Science and Engineering, Academic Press, Boston, Mass, USA, 1992.

[8] G. Toader and J. Sándor, "Inequalities for general integral means," Journal of Inequalities in Pure and Applied Mathematics, vol. 7, no. 1, article 13, pp. 1-5, 2006.

[9] K. B. Stolarsky, "Generalizations of the logarithmic mean," Mathematics Magazine, vol. 48, pp. 87-92, 1975.

[10] E. B. Leach and M. C. Sholander, "Extended mean values," The American Mathematical Monthly, vol. 85 , no. 2, pp. 84-90, 1978.

[11] F. Qi, "A note on Schur-convexity of extended mean values," The Rocky Mountain Journal of Mathematics, vol. 35, no. 5, pp. 1787-1793, 2005.

[12] J. Sándor, “The Schur-convexity of Stolarsky and Gini means," Banach Journal of Mathematical Analysis, vol. 1, no. 2, pp. 212-215, 2007.

[13] Y. Chu and X. Zhang, "Necessary and sufficient conditions such that extended mean values are Schurconvex or Schur-concave," Journal of Mathematics of Kyoto University, vol. 48, no. 1, pp. 229-238, 2008.

[14] D.-M. Ji and H.-N. Shi, "Schur convexity and Schur-geometrically concavity of generalized exponent mean," RGMIA Research Report Collection, vol. 11, no. 4, article 2, 2008.

[15] J. Pečarić and G. Roqia, "Generalization of Stolarsky type means," to appear in Journal of Inequalities and Applications.

[16] M. Anwar and J. Pečarić, "New means of Cauchy's type," Journal of Inequalities and Applications, vol. 2008, Article ID 163202, 10 pages, 2008.

[17] A. W. Roberts and D. E. Varberg, Convex Functions, vol. 5 of Pure and Applied Mathematics, Academic Press, New York, NY, USA, 1973.

[18] P. C. Hammer, "The midpoint method of numerical integration," Mathematics Magazine, vol. 31, pp. 193-195, 1958.

[19] P. S. Bullen, "Error estimates for some elementary quadrature rules," Univerzitet u Beogradu. Publikacije Elektrotehničkog Fakulteta. Serija Matematika i Fizika, no. 602-633, pp. 97-103, 1978.

[20] S. S. Dragomir, D. S. Milošević, and J. Sàndor, “On some refinements of Hadamard's inequalities and applications," Univerzitet u Beogradu. Publikacija Elektrotehničkog Fakulteta. Serija Matematika, vol. 4, pp. 21-24, 1993.

[21] S. S. Dragomir and C. E. M. Pearce, Selected Topics on Hermite-Hadamard Inequalities and Applications, RGMIA Monographs, Victoria University, 2000, http://rgmia.vu.edu.au/monographs/.

[22] G.-S. Yang and M.-C. Hong, "A note on Hadamard's inequality," Tamkang Journal of Mathematics, vol. 28, no. 1, pp. 33-37, 1997.

[23] H.-N. Shi, "Schur-convex functions related to Hadamard-type inequalities," Journal of Mathematical Inequalities, vol. 1, no. 1, pp. 127-136, 2007.

[24] V. Čuljak and J. Pečarić, "Schur-convexity of Čebišev functional," to appear in Mathematical Inequalities $\mathcal{E}$ Applications.

[25] V. Čuljak, "A remark on Schur-convexity of the mean of a convex function," to appear in Mathematical Inequalities $\mathcal{E}$ Applications.

[26] S. S. Dragomir, "Two mappings in connection to Hadamard's inequalities," Journal of Mathematical Analysis and Applications, vol. 167, no. 1, pp. 49-56, 1992.

[27] Y. Chu, G. Wang, and X. Zhang, "Schur convexity and Hadamard's inequality," Mathematical Inequalities E Applications, vol. 13, no. 4, pp. 725-731, 2010.

[28] A. Aglić Aljinović and J. Pečarić, "On some Ostrowski type inequalities via Montgomery identity and Taylor's formula," Tamkang Journal of Mathematics, vol. 36, no. 3, pp. 199-218, 2005.

[29] X.-L. Cheng and J. Sun, "A note on the perturbed trapezoid inequality," Journal of Inequalities in Pure and Applied Mathematics, vol. 3, no. 2, article 29, pp. 1-7, 2002.

[30] D. Zwick, "A divided difference inequality for $n$-convex functions," Journal of Mathematical Analysis and Applications, vol. 104, no. 2, pp. 435-436, 1984.

[31] J. E. Pečarić, "An inequality for 3-convex functions," Journal of Mathematical Analysis and Applications, vol. 90, no. 1, pp. 213-218, 1982.

[32] R. Farwig and D. Zwick, "Some divided difference inequalities for $n$-convex functions," Journal of Mathematical Analysis and Applications, vol. 108, no. 2, pp. 430-437, 1985. 
[33] J. E. Pečarić and D. Zwick, "n-convexity and majorization," The Rocky Mountain Journal of Mathematics, vol. 19, no. 1, pp. 303-311, 1989.

[34] M. Merkle, "Conditions for convexity of a derivative and some applications to the gamma function," Aequationes Mathematicae, vol. 55, no. 3, pp. 273-280, 1998.

[35] Z.-H. Yang, "On the log-convexity of two-parameter homogeneous functions," Mathematical Inequalities $\mathcal{E}$ Applications, vol. 10, no. 3, pp. 499-516, 2007.

[36] H.-N. Shi, Y.-M. Jiang, and W.-D. Jiang, "Schur-convexity and Schur-geometrically concavity of Gini means," Computers E Mathematics with Applications, vol. 57, no. 2, pp. 266-274, 2009.

[37] A. Witkowski, "On Schur-nonconvexity of Gini means," RGMIA Research Report Collection, vol. 12, no. 2, article 10, 2009.

[38] A. Witkowski, "On two- and four-parameter families," RGMIA Research Report Collection, vol. 12, no. 1, article 3, 2009.

[39] Z.-H. Yang, "On the monotonicity and log-convexity of a four-parameter homogeneous mean," Journal of Inequalities and Applications, vol. 2008, Article ID 149286, 12 pages, 2008. 